\title{
Social connectedness is a protective factor against short-term suicide attempts (post discharge) in school children
}

\author{
By Jessica K. Edwards
}

Researchers in the USA have performed a multi-site, prospective analysis of $>2,000$ adolescents aged $12-17$ years to try to determine the short-term predictors of suicide attempts within 3-months of an emergency department visit. The study population was enriched to include a high proportion of adolescents at risk for suicide attempts. At baseline, $>50 \%$ of the study's follow-up sample reported a lifetime history of suicidal ideation (SI) and $\sim 40 \%$ reported a lifetime history of suicidal behaviour: $4.9 \%$ of the follow-up cohort made a suicide attempt between enrolment and 3-month follow-up. Multivariate analyses identified numerous predictors of short-term suicide attempts in this sample, as well as in four critically important subgroups defined by sex and the presence, or absence, of recent suicidal thoughts. Notably, school or social connectedness emerged as a key protective factor for the total follow-up sample and several subgroups of adolescents, including adolescents who did not report suicidal thoughts at baseline, and adolescent females. This key predictor was not significant for adolescent males.

Consistent with these findings, a growing body of research ${ }^{1}$ suggests that higher levels of school connectedness are associated with a lower prevalence of suicidal behaviours in general school samples, high risk adolescents, and sexual minority adolescents. ${ }^{2}$ The researchers propose, therefore, that social and school connectedness might be an important target for suicide attempt risk assessment and preventive intervention.

King, C. et al. (2019), Predicting 3-month risk for adolescent suicide attempts among paediatric emergency department patients. J. Child Psychol. Psychiatr. doi:10.1111/jcpp.13087

\section{References}

${ }^{1}$ Gunn, J. F., Goldstein, S. E. and Gager, C. T. (2018). A longitudinal examination of social connectedness and suicidal thoughts and behaviours among adolescents. Child Adolesc. Ment. Health, 23(4), 341-350. doi:10.1111/camh.12281

${ }^{2}$ Marraccini, M. E. and Brier, Z. M. (2017).

School connectedness and suicidal thoughts and behaviours: systematic meta-analysis. Sch. Psychol. Q., 32(1), 5-21. doi: 10.1037/spq0000192.

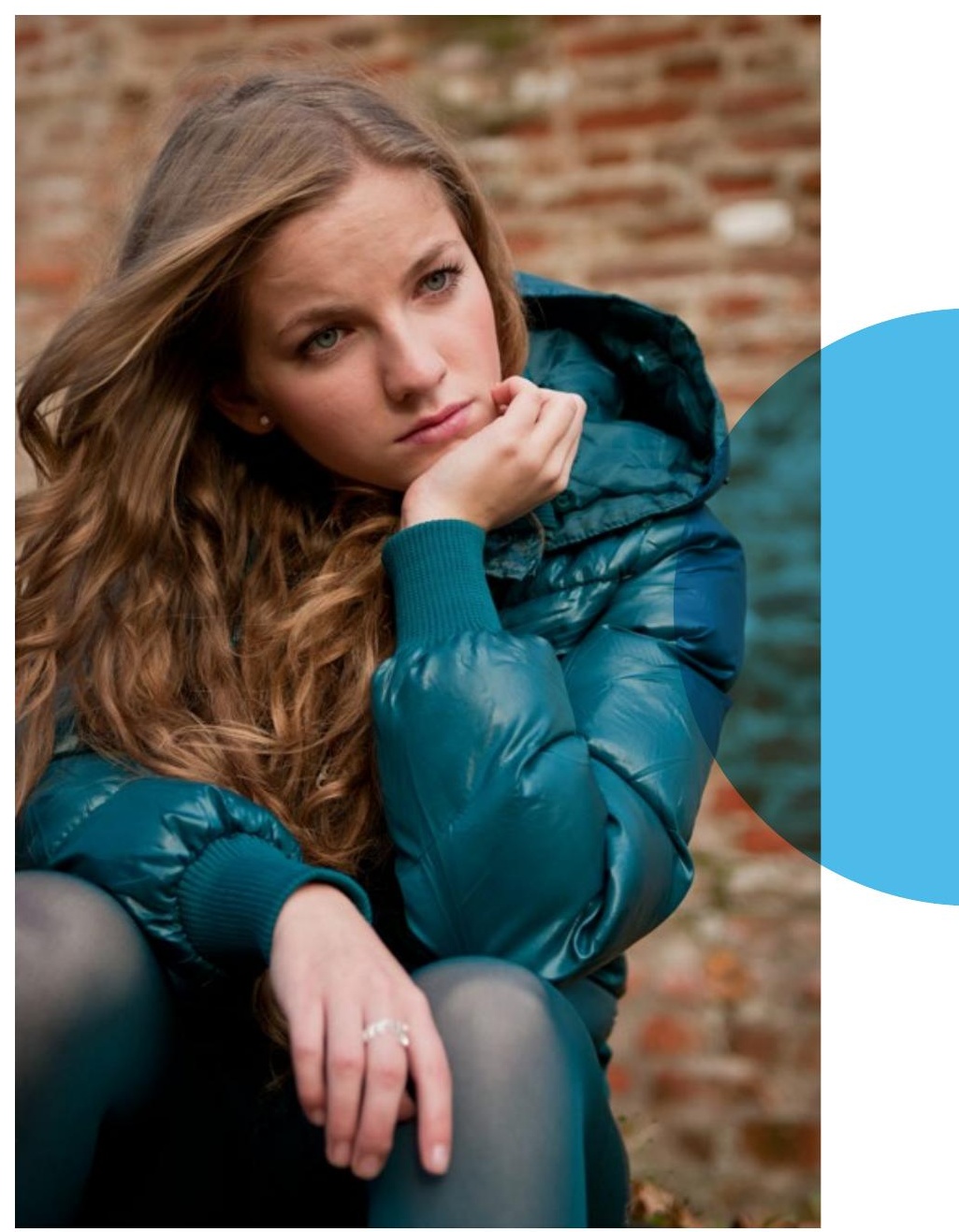

